\title{
Wide-band error-free wavelength conversion based on continuous-wave-triggered supercontinuum
}

\author{
Xing Xu, Chi Zhang, T. I. Yuk, Kevin K. Tsia, and Kenneth K. Y. Wong* \\ Photonic System Research Laboratory, Department of Electrical and Electronic Engineering, \\ The University of Hong Kong, Pokfulam Road, Hong Kong. \\ *Email: kywong@eee.hku.hk
}

\begin{abstract}
We demonstrate a wavelength converter based on $\mathrm{CW}$-triggered picosecond supercontinuum (SC), with significantly enhanced spectrum over 300-nm. While error-free operations are obtained for wavelength converted signals from 1510 to $1615 \mathrm{~nm}$.

(C) 2012 Optical Society of America

OCIS codes: (190.0190) Nonlinear optics; (320.6629) Supercontinuum generation; (230.7405) Wavelength conversion devices.
\end{abstract}

\section{Introduction}

Supercontinuum (SC), with its ultrabroadband spectrum generated from narrow-band pulses, is the potential light source for a wide range of applications, among which includes wavelength-division multiplexing (WDM) in optical communications, ultrashort pulse generation, wavelength conversion, optical frequency metrology, and optical coherence tomography (OCT) [1]. One primary scheme for ultrabroadband SC generation is based on soliton fission, which happens when launching intense pulsed pump into the nonlinear optical fiber at its zero-dispersion wavelength (ZDW) [2]. However, as being initiated by modulation instability (MI), and spontaneously grows from noise, the soliton fission is in a random manner. Although rendering an ultrabroadband spectrum, the inherent incoherence and pulse-to-pulse intensity fluctuation with the generated SC limit its application [3]. This is particularly severe for wider input pulsewidth: picosecond or nanosecond [1]. The dilemma can be eased by introducing an weak continuous-wave (CW) triggering signal [4]. Instead of the noise, the MI growth process can be initiated by the controllable trigger. Comparing to some former schemes [5,6], this method provides a simple and effective solution to enhance both the covering range and the pulse stability of the generated SC.

These enhancements mold the CW-triggered SC into a promising candidate for the wide range wavelength converter. In some former experiments [7-9], which characterized by narrow SC spectrum bandwidth (tens of nanometer), only limited range of wavelength conversion could be achieved. While in this paper, we demonstrated a wavelength converter utilizing the $\mathrm{CW}$-triggered picosecond SC scheme with the input of 10-Gb/s pulsed pump and 7-ps pulsewidth. Error-free (2.6 to 3.9-dB power penalty at the bit-error rate (BER) of $10^{-9}$ ) operations were attained for over 100-nm spectrum range (S, C and L-band), while the whole SC spectrum was increased to over $300 \mathrm{~nm}$.

\section{Principle and Experimental Setup}

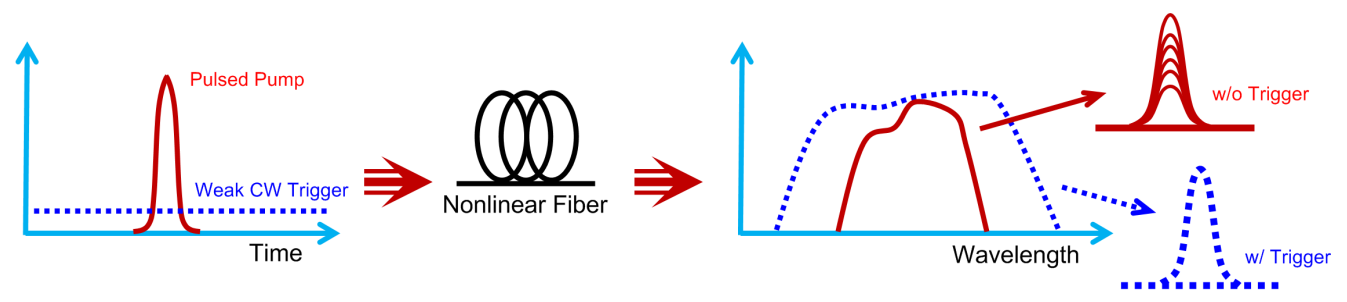

Fig. 1. Principle of the weak CW-triggered SC scheme (solid line: only pulsed pump; dotted line: after injecting the weak CW trigger).

The principle of the CW-triggered picosecond SC is illustrated in Fig. 1 above. It is realized by an external weak $\mathrm{CW}$ trigger, co-propagating inside the fiber with the picosecond pulsed pump. The generated SC spectrum can then be significantly enhanced when the wavelength of the trigger located at the MI gain peak [2]. As previously explained in the introduction, the MI growth here is initiated by the trigger, rather than the noise, which leads to 
the stabilized soliton fission during the SC generation process. As a result, in addition to the spectrum broadening, the pulse-to-pulse instability is also improved, which can be visualized by the clear open eye diagram comparing to the non-triggered case (rightmost portion of Fig. 1).

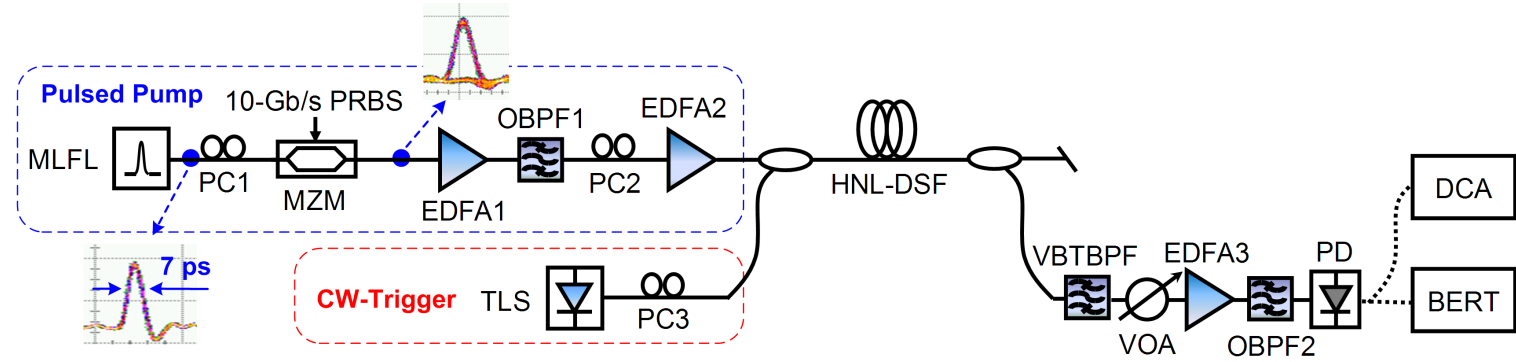

Fig. 2. Experimental setup for the wide range wavelength conversion based on seeded-SC scheme. MLFL: mode locked fiber laser, MZM: Mach-Zehnder modulator, VBTBPF: variable bandwidth tunable band pass filter, VOA: variable optical attenuator, PD: photodiode. (other abbreviations are defined in the text)

The experimental setup is shown in Fig. 2 above. The pulsed pump, located at $1554.77 \mathrm{~nm}$, were generated from a mode-locked fiber laser source (MLFL) with the pulsewidth of 7 ps and then intensity-modulated by a Mach-Zehnder modulator (MZM) driven by $10-\mathrm{Gb} / \mathrm{s} 2^{31}-1$ pseudorandom binary sequence (PRBS). While the polarization controller (PC1) was utilized to align the polarization of the pulse with the transmission axis of the MZM. Two erbium-doped fiber amplifiers (EDFA) were applied to amplify the average pump power upto 365 $\mathrm{mW}(\sim 10.43 \mathrm{~W}$ peak power) before the highly-nonlinear dispersion-shifted fiber (HNL-DSF), which has the length $L=150 \mathrm{~m}$, ZDW $\lambda_{0}=1554.7 \mathrm{~nm}$ and nonlinear coefficient $\gamma=14 \mathrm{~W}^{-1} \mathrm{~km}^{-1}$. The optical bandpass filter (OBPF), with 3-nm bandwidth, between the two EDFAs was used to minimize the amplified spontaneous emission (ASE) noise level from EDFA1. On the triggering signal branch, one CW tunable laser source (TLS), wavelength at $1613 \mathrm{~nm}$, was introduced. The power level of the triggering signal is more than $40-\mathrm{dB}$ lower than the pump. After combining with a WDM coupler, the pulsed pump and the weak triggering signal were launched together into the HNL-DSF. At the fiber output, after removing the residual pump the wavelength converted pulsed signal was filtered out by a variable bandwidth tunable bandpass filter (VBTBPF). After preamplifier (EDFA3), the eye diagrams and the BER of the converted signal were then measured by digital communication analyzer (DCA) and BER tester (BERT), respectively.

\section{Experimental Results and Discussion}

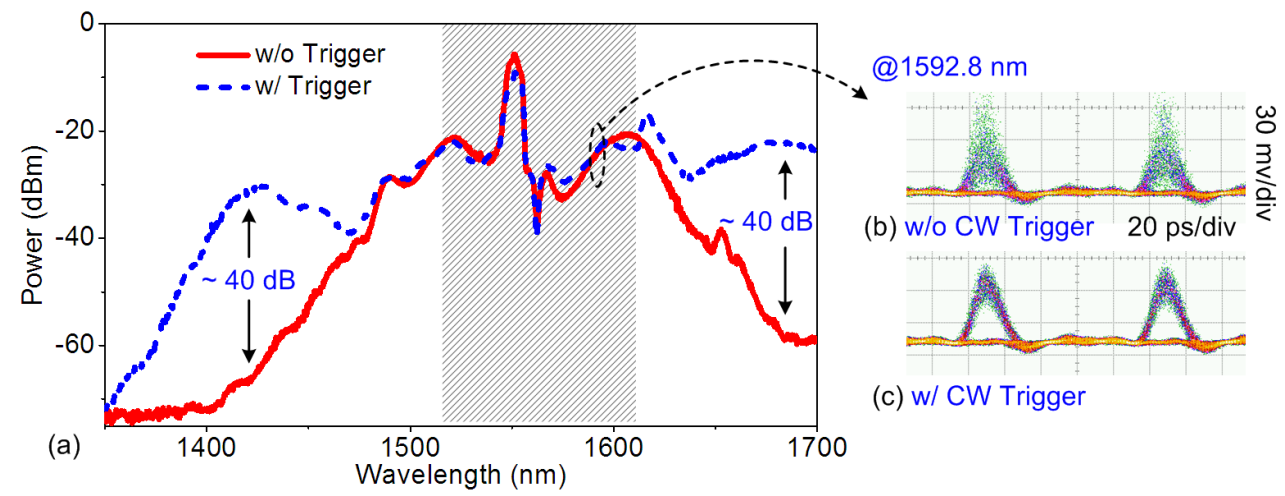

Fig. 3. (a) Supercontinuum spectra after HNL-DSF: without (red solid line) and with (blue dotted line) triggering signal; (b) and (c): eye diagrams @1592.8 nm without and with triggering signal (OSA resolution bandwidth: $0.06 \mathrm{~nm}$ ).

The optical spectra, at the output of HNL-DSF, are shown in Fig. 3. The dip around $1560 \mathrm{~nm}$ in Fig. 3(a) was due to the pump filtering after the WDM coupler. Clearly observed from Fig. 3(a), the original spectrum of the SC was greatly modified after introducing the weak triggering signal, with significant enhancement at some wavelength regions (1380 nm to $1470 \mathrm{~nm}, 1630 \mathrm{~nm}$ to $1700 \mathrm{~nm}$ and beyond). While the largest power enhancement took place around $1400 \mathrm{~nm}$ and $1700 \mathrm{~nm}$ with nearly 40-dB increase comparing to the non-triggering case. In order to investigate the quality of the wavelength conversion based on our CW-triggered SC scheme, different WDM 
couplers were used to slice out the shadowed area in Fig. 3(a) from S-band (1510 nm) to L-band (1615 nm), which covered over $100 \mathrm{~nm}$. Fg. 3(b) and (c) show the wavelength converted signal at $1592.8 \mathrm{~nm}$ originated from pulsed pump, and the bandwidth of the VBTBPF was $3.2 \mathrm{~nm}$. The improvement with triggering is obvious from the eye diagram.
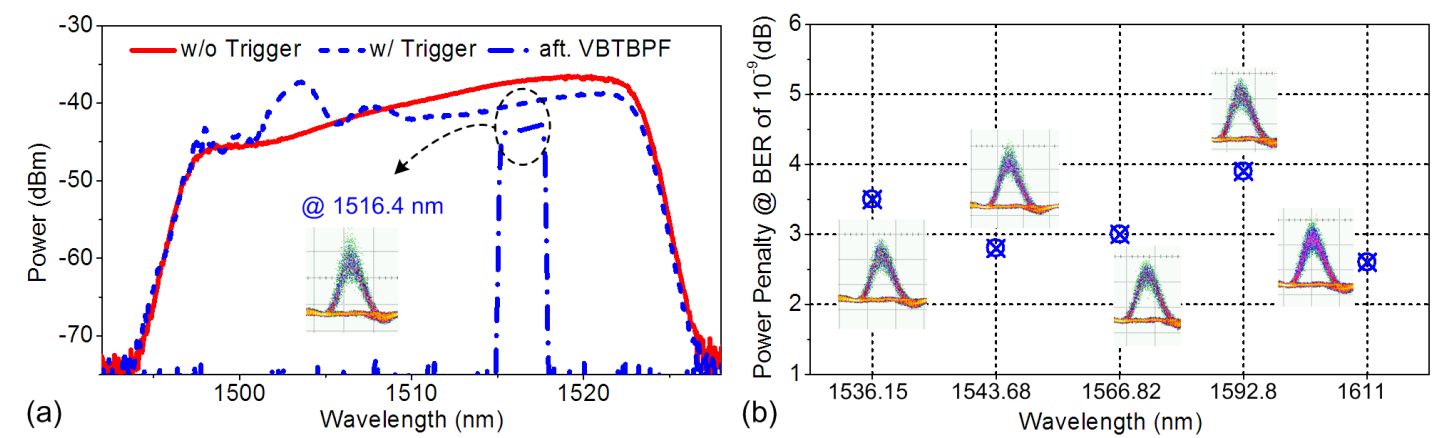

Fig. 4. (a) Part of the S band spectrum filtered out by WDM coupler: without triggering (red solid), with triggering (blue dash) and at the VBTBPF output @ 1516.4 nm (blue dash dot); (b) Measured power penalties at the BER of $10^{-9}$ and eye diagrams for different wavelengths in C-band and L-band (OSA resolution bandwidth: $0.06 \mathrm{~nm}$ ).

In addition to the one at $1592.8 \mathrm{~nm}$, several more wavelengths (C-band: 1536.15 and $1543.68 \mathrm{~nm}$; L-band: 1566.82 and $1611 \mathrm{~nm}$ ) were measured across the $100 \mathrm{~nm}$ spectrum as shown in the shadowed area of Fig. 3(a) while keeping the same filter bandwidth. With the exception of S-band (Fig. 4(a)), for which only the eyediagram at $1516.4 \mathrm{~nm}$ was measured, all the recorded eye diagrams and their corresponding BER at $10^{-9}$ are shown in Fig. 4(b). Stability improvement can be observed from the eye diagrams for all measured wavelengths. Error-free operations were achieved with 2.6 to $3.9-\mathrm{dB}$ power penalty at the BER of $10^{-9}$. With proper filtering and detection equipment, it will also be interesting to further explore the red-shifted (around $1400 \mathrm{~nm}$ ) and blue-shifted (around $1700 \mathrm{~nm}$ ) region of the triggered SC spectrum.

\section{Conclusion}

We have demonstrated a wavelength converter based on the CW-triggered picosecond SC scheme. Comparing to the original non-triggered case, the SC spectrum was significantly enhanced to nearly doubled bandwidth (wider than $300 \mathrm{~nm}$ ). With the triggering process, clear and widely open eye diagrams have been obtained for all wavelength points, from 1510 to $1615 \mathrm{~nm}$. While less than or equal to 3.9-dB power penalty at the BER of $10^{-9}$ were achieved for C-band and L-band signals. The proposed scheme provides a wide covering range solution for wavelength conversion. With narrower pulsewidth, it can be further extended to $40-\mathrm{Gb} / \mathrm{s}$ or even higher data rate.

\section{Acknowledgment}

The work described in this paper was partially supported by the grant from the Research Grants Council of the Hong Kong Special Administrative Region, China (Projects No. HKU 7179/08E, HKU 7183/09E and HKU $7175 / 10 \mathrm{E})$.

\section{References}

1. J.M. Dudley et al., "Supercontinuum generation in photonic crystal fiber," Rev. Mod. Phys., 78, 1135, (2006)

2. J. Herrmann et al., "Experimental Evidence for Supercontinuum Generation by Fission of Higher-Order Solitons in Photonic Fibers," Phys. Rev. Lett., 88, 173901, (2002).

3. G. Genty et al., "Fiber supercontinuum sources (Invited)," J. Opt. Soc. Am. B, 24, 1771, (2007).

4. K.K.Y. Cheung et al., "Manipulating supercontinuum generation by minute continuous wave," Opt. Lett., 36, 160, (2011).

5. D.R. Solli et al., "Active Control of Rogue Waves for Stimulated Supercontinuum Generation,” Phys. Rev. Lett., 101, 233902, (2008).

6. P.M. Moselund et al., "Back-seeding of higher order gain processes in picosecond supercontinuumgeneration," Opt. Express, 16, 11954, (2008).

7. M. Scaffardi et al., "Implementation of a tunable $160 \mathrm{~Gb} / \mathrm{s}$ wavelength multi-converter based on supercontinuum in a highly nonlinear fibre," in European Conference on Optical Communications, ECOC, pp. 1-2, (2006).

8. O.F. Yilmaz et al., "Tunable N-fold multicasting and pulsewidth of $40 \mathrm{~Gb} / \mathrm{s}$ channels by variable periodic slicing of a supercontinuum," in Conference on Lasers and Electro-Optics and Laser Science, CLEO/QELS, pp. 1-2, (2009).

9. S. Oshiba et al., "All-optical arbitrary wavelength conversion with signal regeneration based on slicing of supercontinuum spectrum," in IEEE/LEOS Winter Topicals Meeting Series, 256, (2009). 